\title{
Research on Automatic Temperature Adjusting and Controlling System of Ginseng Drying
}

\author{
Ji Li ${ }^{\mathrm{a}}$, Jian $\mathrm{Li}^{\mathrm{b}}$, Hongjun $\mathrm{Gu}^{\mathrm{c}}$, He Gong ${ }^{\mathrm{d}}$ and Shijun $\mathrm{Li}^{\mathrm{e}}$ \\ Jilin Agricultural University, Changchun 130118, Jilin, China \\ a735485328@qq.com, b16289144@qq.com, '330837495@qq.com, d29878671@qq.com, \\ Isj0883@sina.com
}

\section{Keywords: Ginseng drying; Temperature; Humidity; Automatic control}

\begin{abstract}
Drying is an important part in the process of producing ginseng, and it is an important means of processing and storage. At present, the drying system based on the dryer can not control the drying environment intelligently, and it has a great influence on the intrinsic quality, smell and water content of the ginseng. Through the analysis and research of the temperature control of existing ginseng processing equipment, this paper puts forward a kind of intelligent temperature controlling system which consists of wireless multi-point temperature and humidity collection, alarm displaying and intelligent controlling. This system has a great practical significance in solving the demand contradiction of the drying temperature of the ginseng, protecting the drying quality of the ginseng, improving the drying efficiency of the ginseng, reducing the labor force, saving energy and reducing emission.
\end{abstract}

\section{Introduction}

As we all known, the ginseng has been regarded as the king of herbs since the ancient times. It is the most precious herb in the medical library at all times and in all countries [1]. It has been admitted that the ginseng is the best tonic by the public. We have utilized the ginseng for many years, but the number of the ginseng, as a kind of natural and green tonic, becomes less and less at present. China is the country that has a large yield of the ginseng. The proportion of the ginseng that is produced in China is about $85 \%$ of the total yield in the world.

Ginseng drying is mainly achieved by the dryer at present. However, ginseng drying can not be achieved in batch so that the production efficiency is low [3]. The dryer can just control a single temperature so that the quality of ginseng will be decreased [4]. In addition, the power consumption of the dryer is very large so that energy consumption is very large. With the modern development of facility agriculture, the microprocessor, mainly referring to single chip microcomputers, plays an important role in monitoring and controlling, especially in temperature controlling [5-7]. SCMs have many characteristics including high reliability, short developing period, small volume, low manufacturing price and strong functions. Based on the actual requirements and technology of similar researches, a new, intelligent and environmental plan, called automatic temperature adjusting and controlling system of ginseng drying, has been designed independently. SCMs can control temperature intelligently in the automatic temperature controlling system of this research project [8]. The sensor detection technology has been used to collect information parameters of the temperature. The relay controlling technology has been used to control the temperature. The quality of products has been improved greatly. Meanwhile, problems of the dryer have been solved.

\section{The Overall Design of the System}

The automatic controlling system of the temperature and the humidity in the glass house where ginseng can be dried is composed of monitoring temperature and humidity, controlling intelligently and the human-computer interaction. The function of monitoring temperature and humidity refers to the temperature and humidity in the glass houses need to be collected at many points and in real-time, and then the collected data will be transferred to main processing units of the SCM by wireless to control the 
temperature and the humidity intelligently [9]. The function of intelligent control refers to data that is transmitted by the sensor should compare with the setting value. The SCM system can control on-off of the relay by instructions and the on-off of relays can control the on-off of thermal control equipment and dehumidifying equipment. It is difficult to monitor the humidity in the domestic at present, so the humidity can be just displayed in this system. People can guarantee the humidity in the house to meet the required requirement for ventilation and air exchange according to the humidity and air condition transmitted. The function of human-computer interaction mainly refers to operators can input the required and proper temperature environment by the keyboard so that the current temperature, the humidity in the house, the current date and the time can be displayed. When the values of the temperature and humidity in the glass house are beyond the corresponding value or the equipment, the sound-light alarm gives the alarm signal. Fig .1 describes the schematic structure of the system.

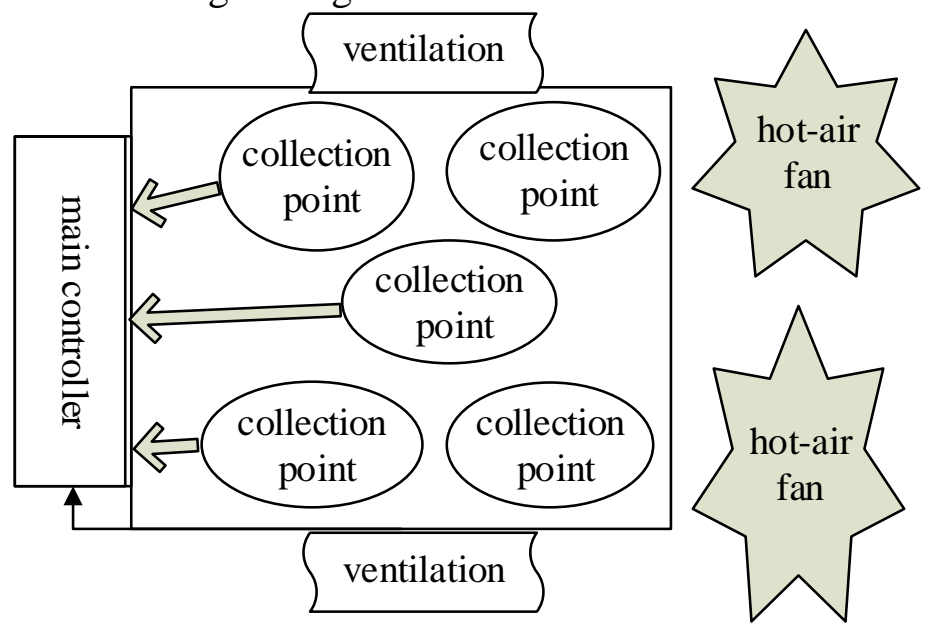

Figure 1. Schematic structure

The Designing of Monitoring and Controlling Devices of SCM. Most of the monitoring and controlling systems in our country depend on imports. The equipment that is introduced from other countries is very expensive and not fit for the local situation during practical application in our country. It is very common that the introduced system will not be used totally or partially after using for a period of time. Considering the above situation, it is necessary to develop a modern monitoring and controlling system that can meet the needs. The following functions should be achieved in the design of monitoring and controlling system of SCM.

The first one is that the system can collect and display the real-time parameters of the temperature and the humidity in the glass house. The temperature and the humidity in the glass house of ginseng drying can be gained by real-time multi-point collection. The collected parameters of the temperature and the humidity will be transmitted by wireless, and then the main controller of SCM will control the temperature and the humidity intelligently. The temperature and the humidity in the glass house of ginseng drying can be displayed in real-time by LCD12864 (Liquid Crystal Display).

The second one is that the system can adjust the environment parameter automatically within a certain range in the drying house according to users' needs. This system can control adjusting mechanism automatically and adjust the environment parameter to the parameter value that is set by operators.

The third one is that the system can be set different controlling parameters according to different seasons and different regions. Managers can set different controlling parameters according to different seasons, regions and crops.

\section{The Designing of Electric Circuit in Automatic Temperature and the Humidity Controlling System of Ginseng Drying in the Glass House}

Based on a large number of relevant information, the environment requirement of ginseng drying can be determined. It is very necessary for the designing of automatic environment controlling system of ginseng, and then the hardware structure of automatic environment controlling system of ginseng can be 
determined. The digital temperature sensor DS18B20 will be used to monitor the temperature. The digital temperature and the humidity sensor DHT11 will be used to monitor the humidity. The data information can be transmitted by the wireless sensing module NRF24L01. STC89C52 SCM can deal with the data, control the system, drive LCD12864 to display and send the order to the outer equipment (hot-air fan and ventilation opening) so that the temperature can be controlled. The software program is edited by the $\mathrm{C}$ programming language. The program designing technology combining the modularization and the structuralization is used in the program designing [10].

The Designing of Electric Circuit of the Temperature and the Humidity Collection. The electric circuit of the temperature and the humidity collection consists of the collecting controller, the temperature sensor, the humidity sensor, the wireless sensor module, power management and the resetting indicator circuit. The main part of the collecting controller is STC89C52 SCM. The temperature is collected by the temperature sensor DS18B20. The humidity is collected by the humidity sensor DHT11. Data information is transmitted by wireless sensor modules NRF24L01. The managing unites of the power supply can transform $220 \mathrm{v}$ alternating current to $5 \mathrm{v}$ direct current that can be used by the SCM and the temperature and humidity sensors. It also can transform $5 \mathrm{v}$ direct current to $3.3 \mathrm{v}$ direct current that can be used by wireless sensor modules. Fig .2 shows the collection point circuit diagram.

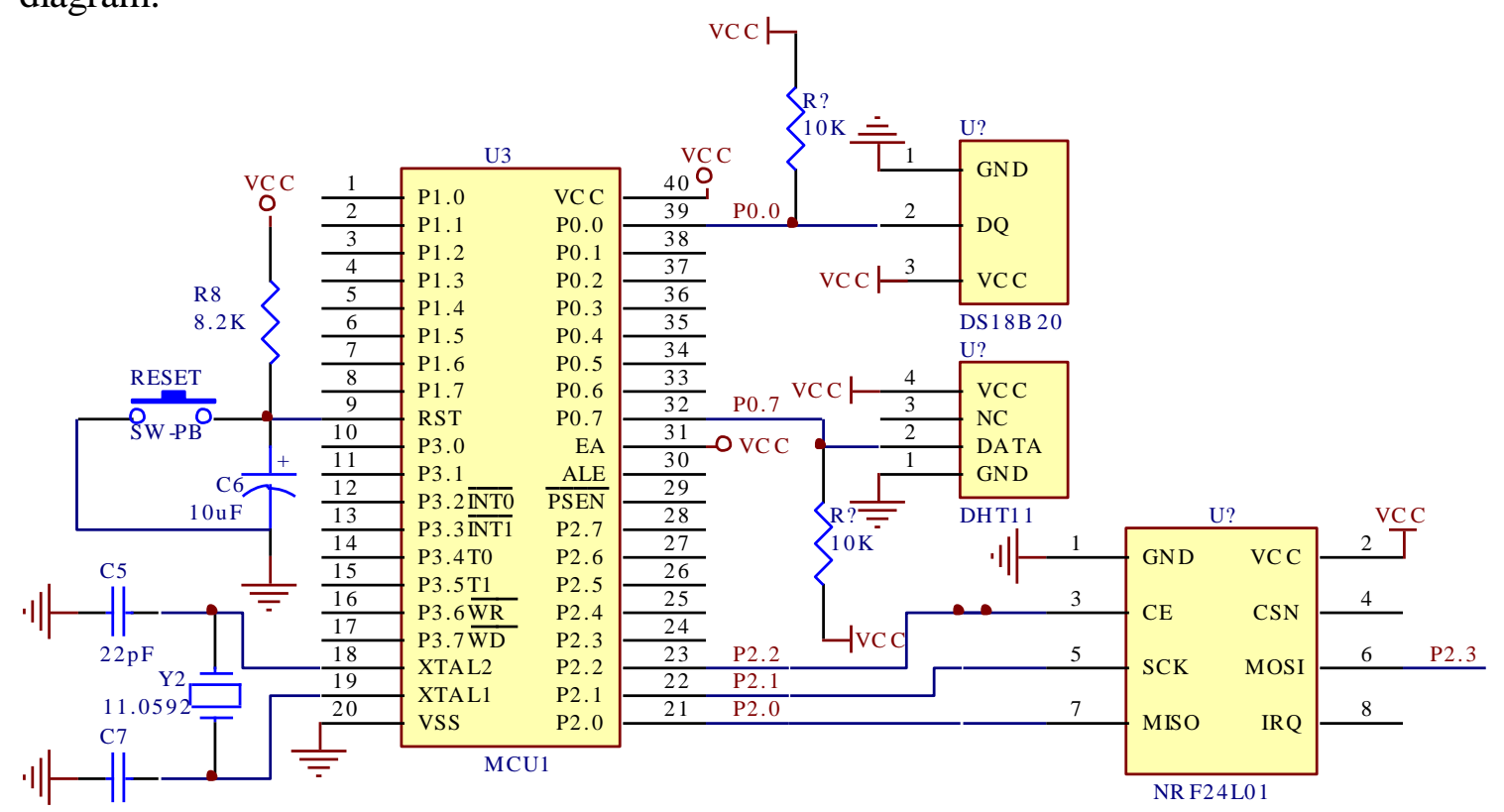

Figure 2 Collection point circuit diagram

The Designing of Electric Circuit of the Human-computer Interaction. The human-computer interaction refers to operators can input the required and proper temperature environment to display the current temperature and the humidity in the house, the current date and time by the keyboard. When the value of the temperature and the humidity in the glass house is beyond the corresponding value or the equipment is out of order, the sound-light alarm will be made by this function. The button part is the $4 \times 4$ keypad structure. There are sixteen buttons, including ten number buttons, four function buttons, one identifying button and one canceling button. The function buttons include the ones used to set days, the ones used to display the temperature, the ones used to display the humidity, the ones used to display data and time, an identifying one and a canceling one. LCD12864 is adopted to display automatically the temperature, the humidity, the current data and the current time in real-time after electrifying. When parameters of the temperature and the humidity in the glass house of ginseng drying are beyond the corresponding value or the equipment is out of order, the alarming information will be displayed. For example, the temperature and the humidity that is collected by the collection controller in a certain bearing of the glass house of ginseng drying are beyond the corresponding value, the main controller will display the information, remind managers that the humidity in this bearing is beyond the corresponding value by LCD12864 and ask the manager whether the system can adjust the humidity or not. When the average temperature and the average humidity that are monitored by the sound-light alarm are beyond 
the alarming value, the buzzer and the luminous diode that are controlled by the main controller will send the sound-light alarming signals. As shown in Fig .3, the main control circuit diagram is designed.

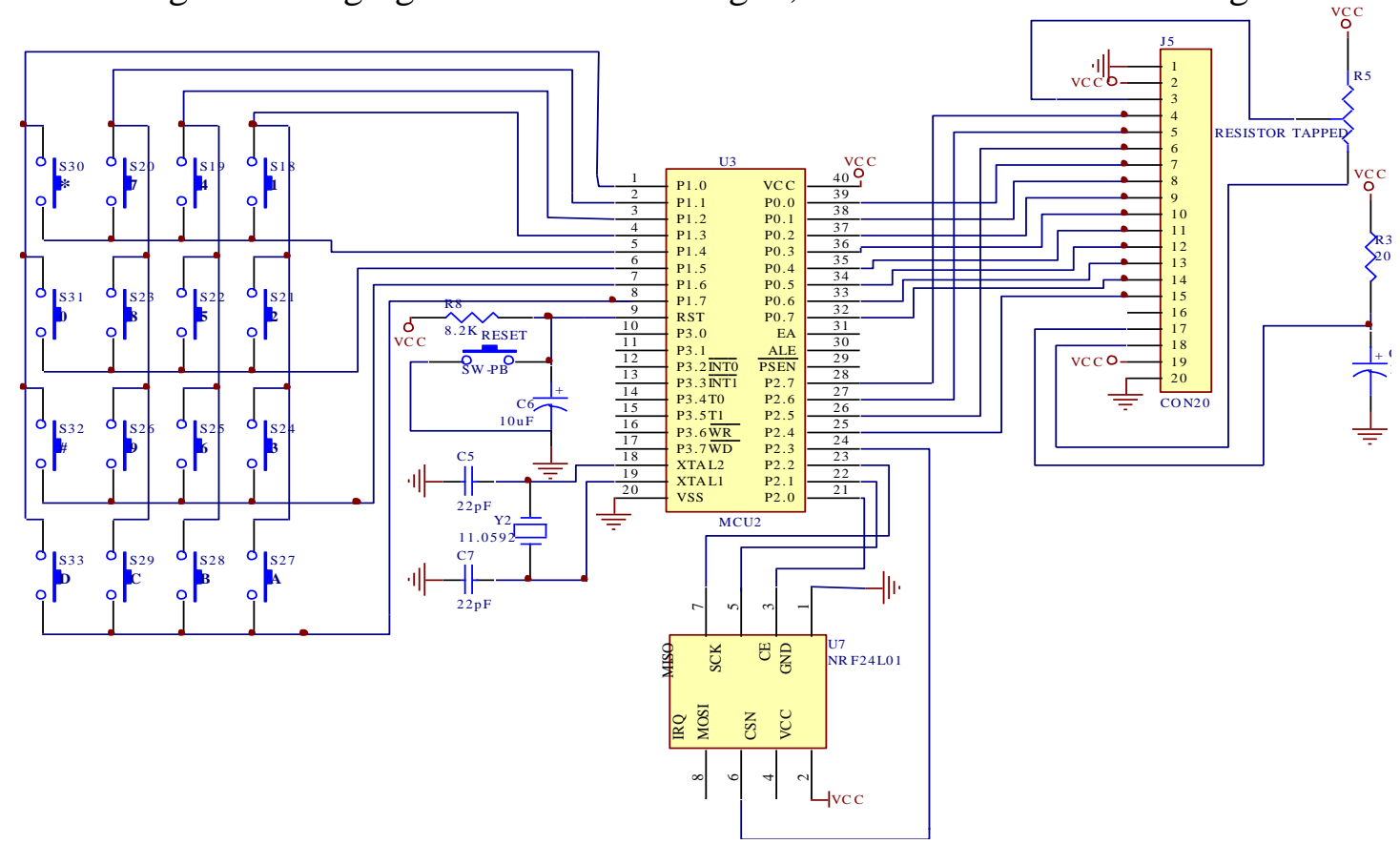

Figure 3. Main control circuit diagram

The Designing of Intelligent Control. The controlling part of the system is used to change the real-time parameters of the temperature and the humidity in the drying glass house. The hot-air fan and the ventilation opening are controlled to open or close by the relay so that the system can control the temperature and the humidity automatically. STC89C52, as the main controller, can control the hot-air fan and the ventilation opening according to the temperature and the humidity in the glass house. The relay controlling circuit and SCM will be separated by photoelectric isolation so that the interference of the relay controlling circuit towards SCM can be avoided. The circuit is shown in the Fig .4.

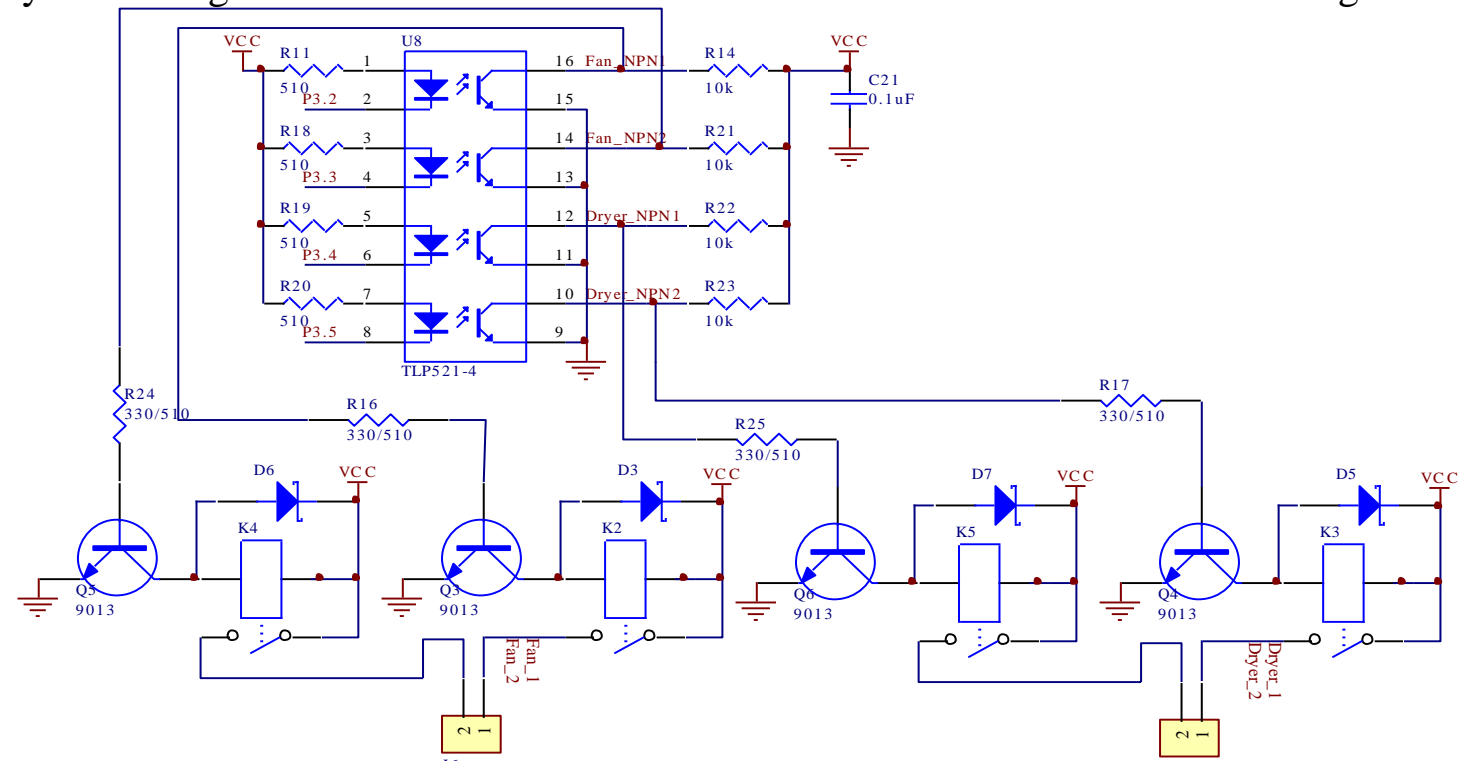

Figure 4. Control circuit diagram

\section{The Software Part of Automatic Temperature and the Humidity Controlling System of Drying}

\section{The Main Program of the System and the Overall Controlling Process.}

The system consists of the sending part and the receiving part. The sending part is composed of the main program module, the communication program module and the monitoring program module of the temperature and the humidity. The receiving part is composed of the main program module, the 
communication program module, the real-time timing program module, the button displaying program module, the storage module and controlling program module. The software part is edited by the $\mathrm{C}$ programming language. The program with modularization is used in the software design. All the modules are independent and connected with each other. The low-level module can be repeatedly used for the high-level module so that the efficiency can be improved and the internal storage can be saved. At the same time, it is convenient to switch, use and maintain the program. Fig .5 shows the main program flow diagram.
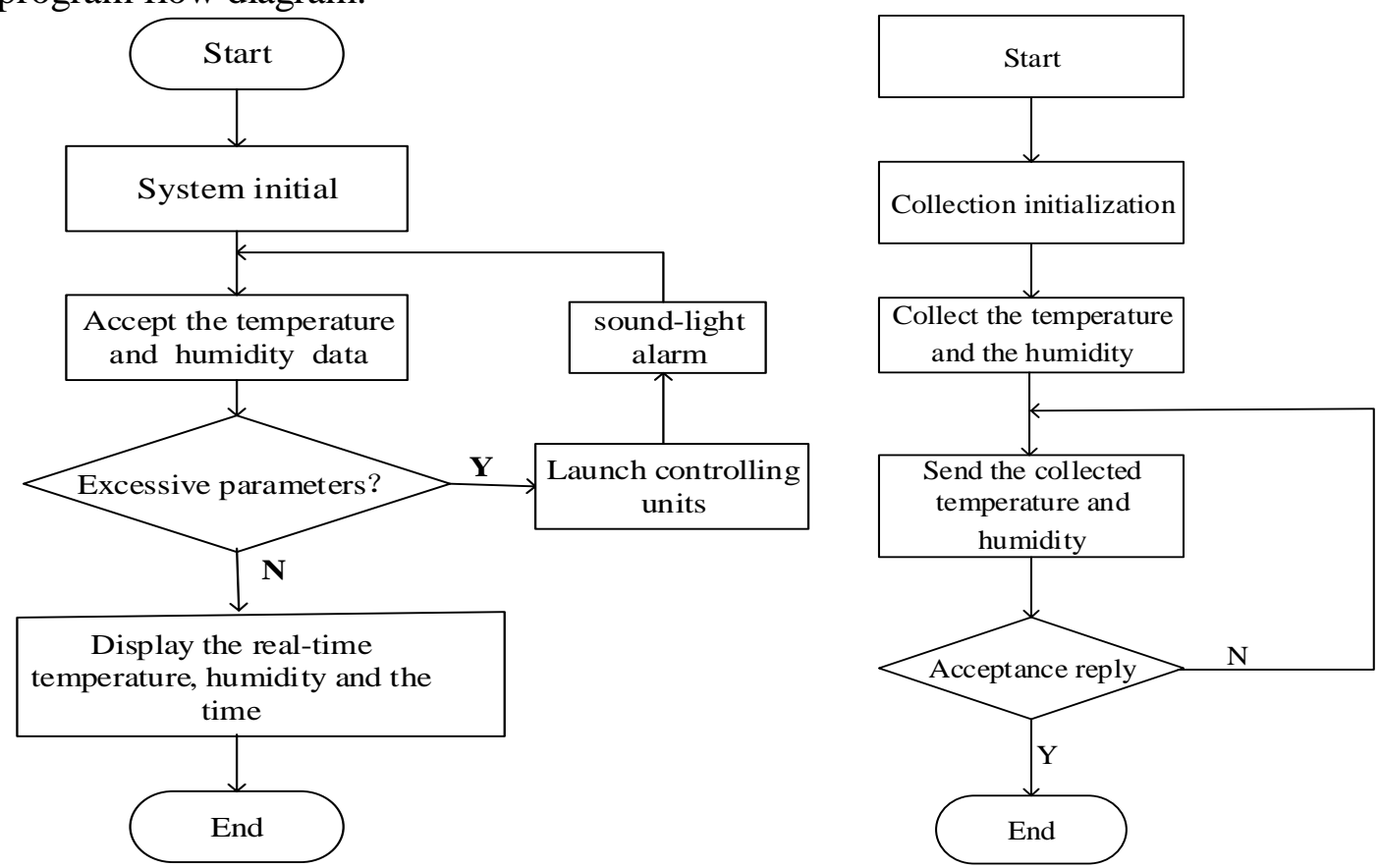

Figure 5. Main program diagram Figure 6. Temperature and humidity acquisition diagram

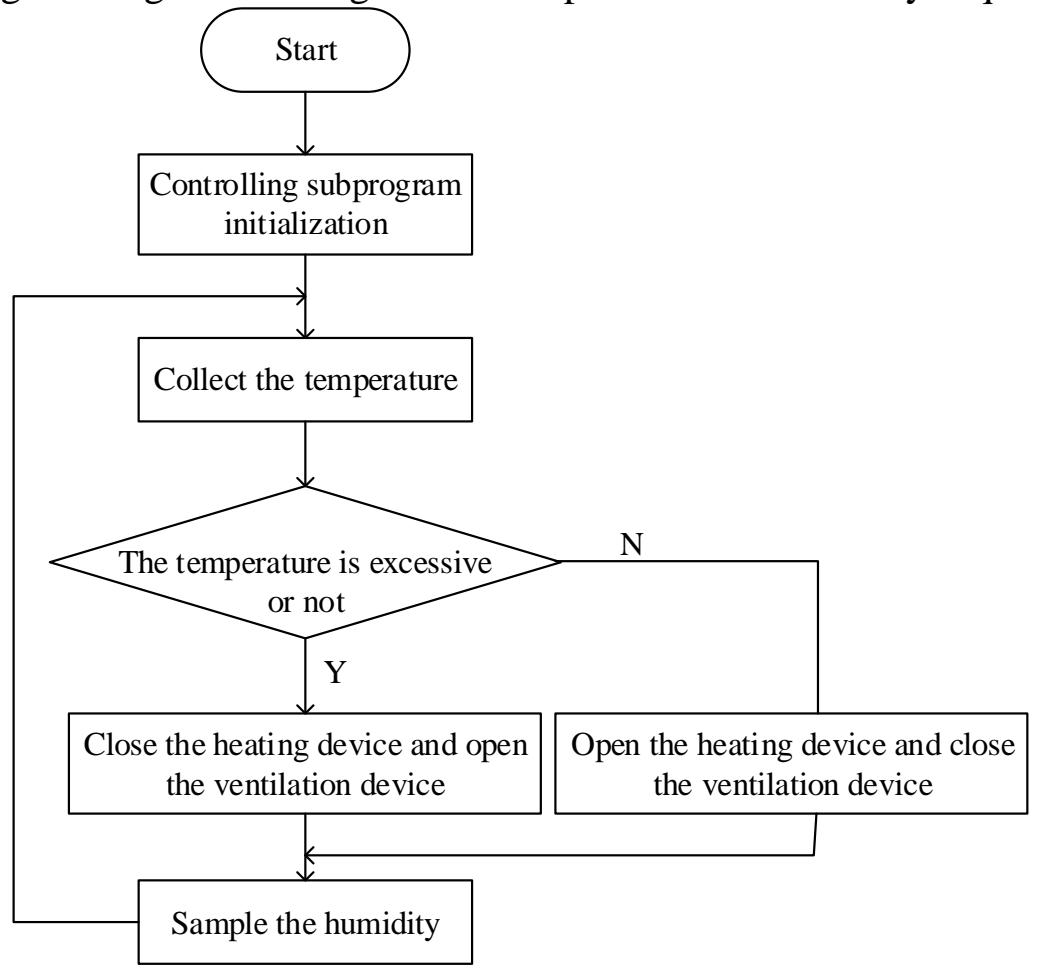

Figure 7. Temperature and humidity control diagram

The Software Process of the Temperature and the Humidity Collection and Control. The running process of the main program module of the temperature and the humidity monitoring and controlling system is shown in Fig .6 and Fig .7. 


\section{Conclusion}

Developing a new type of agriculture by the high and new technology has become a trend of increasing agricultural economy. During this process, there is a highlight that the intelligent monitoring and controlling system in the inner environment should be built to guarantee the high quality products that are provided.

During the process of ginseng drying, the temperature is the key factor that can determine the quality of the ginseng. Based on analyzing the situation of the environment in the glass house, the automatic temperature and the humidity controlling system have been developed according to the requirement of ginseng drying. The STC89C52 is the controlling core of the system. The digital temperature sensor DS18B20 is the temperature measurement component of the system. The system is composed of monitoring the temperature and the humidity, intelligent control and the human-computer interaction. The system can guarantee the quality of dried ginseng, improve efficiency of ginseng drying, reduce manual labor force, save energy and decrease emissions.

\section{Acknowledgements}

The authors wish to express their gratitude to the projects: Jilin Province Economic Structural Adjustment Leading Fund Special Project (No. 2014Y108) and Changchun City Science and Technology Plan Project (No. 14nk029, No. 13KG71), National Natural Science Foundation Project of China (No. 41601454); Agricultural Science and Technology Achievement Transformation Project of Ministry of Science and Technology of China (No. 2014GB2B100021); China Spark Program Project (No. 2015GA660008); Science and Technology Development Plan Project of Jilin Province (No. 20150204058NY, No. 20160519014JH, No. 20140204045NY and No. 20150204058NY); Science and Technology Research Project of "The 13th Five-Year" of The Education Department of Jilin Province (No. JJKH20170296KJ); The Eighth Batch of Engineering Research Center Project of Jilin Province; University Innovation Team Project of the Education Department of Jilin Province, for their generous support of this work.

\section{References}

[1] J.L.Liu: Automatic Instrumentation, Vol.35 (2014), No.6, p.79. (In Chinese)

[2] Z.Z.Fan and Y.C.Liu: Hubei Agricultural Sciences, Vol.54 (2015), No.3, p.705. (In Chinese)

[3] M.Mao: Industrial Instrumentation \& Automation, 2017, No.2, p.84. (In Chinese)

[4] B.X.Gong, B.Y.Sun and X.L.Zhang: Manufacturing Automation, Vol.35 (2013), No.11, p.54. (In Chinese)

[5] Y.K.Zu and M.J.Xu: Journal of Huanggang Normal University, Vol.35 (2015), No.6, p.60. (In Chinese)

[6] F.Yang: Hubei Agricultural Sciences, Vol.55 (2016), No.9, p.2370. (In Chinese)

[7] J.Guan and S.Y.Hu: Journal of Anhui Agri. Sci., Vol.43 (2012), No.23, p.333. (In Chinese)

[8] P.P.Wang and H.L.Huang: Machinery Design \& Manufacture, 2017, No.2, p.40. (In Chinese)

[9] J.Bao: Journal of Agricultural Mechanization Research, 2016, No.2, p.197. (In Chinese)

[10] J. Li, X.Y.Li and J.J.Li, etc: Journal of Jilin an Agricultural Sciences, Vol.40 (2016), No.1, p.100. (In Chinese) 